\title{
Reflection on multilayer mirrors: beam profile and coherence properties
}

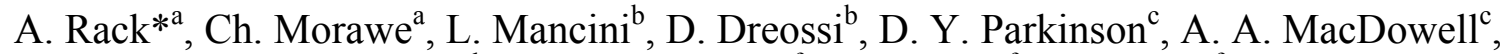

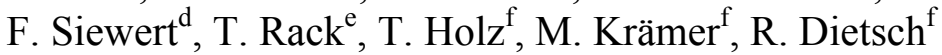 \\ ${ }^{a}$ European Synchrotron Radiation Facility, BP 220, F-38043 Grenoble, France; ${ }^{b}$ Elettra - \\ Sincrotrone Trieste, Area Science Park, I-34149 Basovizza, Italy; 'Advanced Light Source Division, \\ Lawrence Berkeley National Laboratory, Berkeley, California 94720, USA; ${ }^{\mathrm{d}}$ Helmholtz Zentrum \\ Berlin für Materialien und Energie / BESSY-II, Albert-Einstein-Strasse 1, D-12489 Berlin, \\ Germany; ${ }^{\circ}$ Novitom, F-38000 Grenoble, France; ${ }^{f}$ AXO DRESDEN GmbH, Gasanstaltstrasse 8b, \\ D-01237 Dresden, Germany
}

\begin{abstract}
The main advantage of Bragg reflection from a multilayer mirror as a monochromator for hard X-rays, is the higher photon flux density because of the larger spectral bandpass compared with crystal lattice reflection. The main disadvantage lies in the strong modulations of the reflected beam profile. This is a major issue for micro-imaging applications, where multilayer-based monochromators are frequently employed to deliver high photon flux density. A subject of particular interest is the origin of the beam profile modifications, namely the irregular stripe patterns, induced by the reflection on a multilayer. For multilayer coatings in general it is known that the substrate and its surface quality significantly influence the performance of mirrors, as the coating reproduces to a certain degree the roughness and shape of the substrate. This proceedings article reviews recent experiments that indicate potential options for producing wave front-preserving multilayer mirrors, as well as new details on the particular mirrors our group has extensively studied in the past.
\end{abstract}

Keywords: multilayer mirrors, coherence, X-ray monochromator, X-ray imaging, X-ray phase contrast, synchrotron radiation, wave front preservation

\section{INTRODUCTION}

Microimaging techniques are very photon flux demanding. Hence, especially at synchrotron light sources more and more multilayer mirrors are used for monochromatization due to their larger spectral bandpass with respect to crystal-based devices when, for example, microtomography is performed ${ }^{1,2}$. Double-multilayer monochromators are commercially available, including the mirrors and in-vacuum mechanics ${ }^{3-5}$. Despite the increasing demand for wave front-preserving multilayer mirrors, little is known about how the reflection from such a mirror affects the wave front in terms of homogeneity and coherence properties ${ }^{6}$. This knowledge is of crucial importance: (i) the widely applied phase-sensitive imaging techniques are strongly dependent on the wave front properties ${ }^{7}$, (ii) high-definition microtomography requires homogeneous wave fronts to produce artifact-free data sets which are required for a subsequent volume image analysis which takes the technique away from being considered just an academic tool ${ }^{8}$ and (iii) other contrast modalities such as scanning tomography exploiting the powder-diffraction based signal would benefit from the larger photon flux downstream of a multilayer monochromator ${ }^{9}$. Consequently, our group has investigated different multilayers and the corresponding reflected wave fronts at ESRF and different beamlines around the globe. Multilayer mirrors of larger dimension were studied, all of them permanently installed at the ID19 beamline of the European Synchrotron Radiation Facility (ESRF, France) as well as the TopoTomo beamline of the ANKA light source (Germany), ${ }^{4,10}$ : The initial studies were basically confirmed, i.e. that parameters such as d-spacing and the number of bilayers grown have negligible influence on the wave front preservation properties. Of the various multilayers coating tested the $\mathrm{Pd} / \mathrm{B}_{4} \mathrm{C}$ coating yielded the smoothest wave fronts ${ }^{11}$. In order to exclude beamline specific effects the initial mirrors were studied again at other beamlines including at other light sources ${ }^{12}$. A round robin test was initiated between different multilayer deposition

*arack@snafu.de; phone +33 476 88-1781; fax +33 476 88-2785; www.esrf.eu 
facilities which revealed that for textbook-like coatings such as $\mathrm{W} / \mathrm{B}_{4} \mathrm{C}$ a high level of reproducibility in terms of wave front preservation is reached despite the fact that different facilities applied the coating ${ }^{13}$. The corresponding detailed protocol on how to study the wave front preservation properties of a reflective X-ray optical element in a quantitative manner was published ${ }^{14,15}$. Research carried out by other groups in the last years confirmed the theory that the substrate plays a crucial role for the homogeneity of the reflected wave front ${ }^{16}$.

\section{THE BEAMLINE EXPERIENCE}

Modifications of a wave front due to reflection on a multilayer mirror are commonly related to the coherence properties of the impinging wave front ${ }^{6}$. Therefore the beamline layout such as the X-ray optical elements used including the length of the beamline play an important role together with intrinsic properties such as the effective source size. In order to emphasize that the requirements to introduce stripes in a wave front due to reflection from a multilayer are rather relaxed, i.e. basically fulfilled by most beamlines, wave front images have been collected and published in recent years $^{6,11-14}$. The example shown in Fig. 1 was acquired at the SYRMEP beamline of the Elettra light source (Italy) ${ }^{25}$. The bending magnet beamline was operated with a double-crystal monochromator (placed around $16 \mathrm{~m}$ downstream of the source) set to $18 \mathrm{keV}$ X-ray photon energy. Pictures of the wave front were recorded by an indirect detector $(2.2 \mu \mathrm{m}$ effective pixel size) placed around $25 \mathrm{~m}$ downstream of the source. The left picture of Fig. 1 shows the wave front as it is, horizontal stripes are already present due the beamline's beryllium exit window. The right picture of the same figure shows the wave front after additional reflection on a W/ $\mathrm{B}_{4} \mathrm{C}$ multilayer (ESRF deposition facility, $2 \mathrm{~nm} \mathrm{~d}$-spacing, further details on the coating procedure are published $)^{13,17}$. The multilayer introduces an additional stronger stripe pattern. Similar behavior has been observed at other beamlines/other light sources as well. This highlights that modifications of the wave front related to multilayer-reflection are a common problem to all those instruments requiring higher photon flux for phase-sensitive full-field imaging.
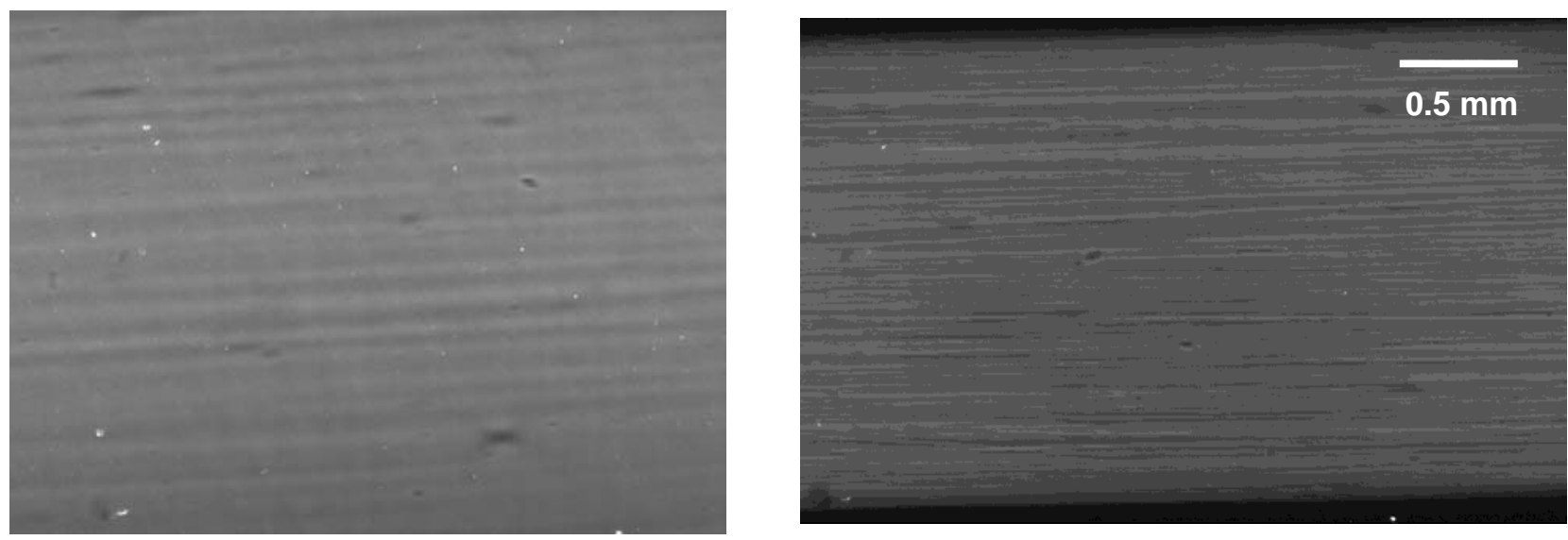

Figure 1. The wave front at the SYRMEP beamline (Elettra light source, Italy). Left: after passing the crystalmonochromator set to transmit $18 \mathrm{keV}$ photon energy, horizontal stripes are present due to the beamline's exit window made from beryllium, right: the wave front after additional reflection on a W/ $\mathrm{B}_{4} \mathrm{C}$ multilayer $(2 \mathrm{~nm} \mathrm{~d}$-spacing, ESRF deposition facility) which introduces an additional more pronounced stripe pattern ${ }^{13}$.

\section{BEAMLINE ROUND ROBIN (CONTINUED)}

A common criticism of the initial study on the wave front preservation properties of multilayer mirrors was that the impact of the beamline properties such as layout, X-ray optical elements or effective source dimensions were not adequately discussed ${ }^{6}$. Hence, beamline round-robins were carried out, i.e. images of wave fronts reported in the initial paper which were recorded at ESRF beamline BM05 were reproduced for example at ESRF beamline ID19 and 32-ID of the Advanced Photon Source (USA) ${ }^{13}$. The pictures of wave fronts acquired after reflection on the $\mathrm{Pd} / \mathrm{B}_{4} \mathrm{C}$ and $\mathrm{Mo} / \mathrm{Si}$ multilayer mirrors $(2.5 \mathrm{~nm}$ d-spacing each) from the original study depicted in Fig. 2 were acquired at the tomography beamline 8.3.2 of the Advanced Light Source (USA) ${ }^{18}$ in 2014. For this experiment the beamline was operated in white beam mode, i.e. the light from the super-bending magnet source was only filtered by a Be exit window and a $1 \mathrm{~mm}$-thick polished Si wafer (21.5 $\mathrm{m}$ from the source) before impinging on the multilayer placed $22 \mathrm{~m}$ downstream of the source. In horizontal deflection geometry due to the available mechanically mounts the mirrors were set to a $\Theta$ angle of 1 degree 
(14.4 keV X-ray photon energy). At a distance of $0.9 \mathrm{~m}$ downstream of the mirror a high resolution detector was positioned to record the images of the wave front $(1.3 \mu \mathrm{m}$ effective pixel size, $5 \times$ Mitutoyo objective (0.14NA) combined with a sCMOS camera type pco.edge by PCO AG (Germany)). The pictures shown were rotated by 90 degree for reproduction as well as a $3 \times 3$ Median filter was applied to reduce high-energy spikes. The top image in Fig. 2 shows the beam as reflected by the Mo/Si multilayer mirror: a vertical stripe pattern is visible, similar to the SYRMEP beamline related to the Be exit window of the beamline. Dark spots are visible as well due to surface damages (i.e. damages due to non-proper storing or handling) or contamination of the mirrors. A low-spatial frequency horizontal stripe pattern can be identified as well, weakly pronounced due to the comparably short distance between multilayer and detector. The bottom picture of the same figure is taken after reflection on the $\mathrm{Pd} / \mathrm{B}_{4} \mathrm{C}$ multilayer mirror. It shows the same vertical stripe pattern as well as dark spots. A horizontal stripe pattern is hardly visible. As previously reported several times, some $\mathrm{Pd} / \mathrm{B}_{4} \mathrm{C}$ multilayer mirrors are capable of supplying a more homogeneous reflected wave front ${ }^{6,13}$.
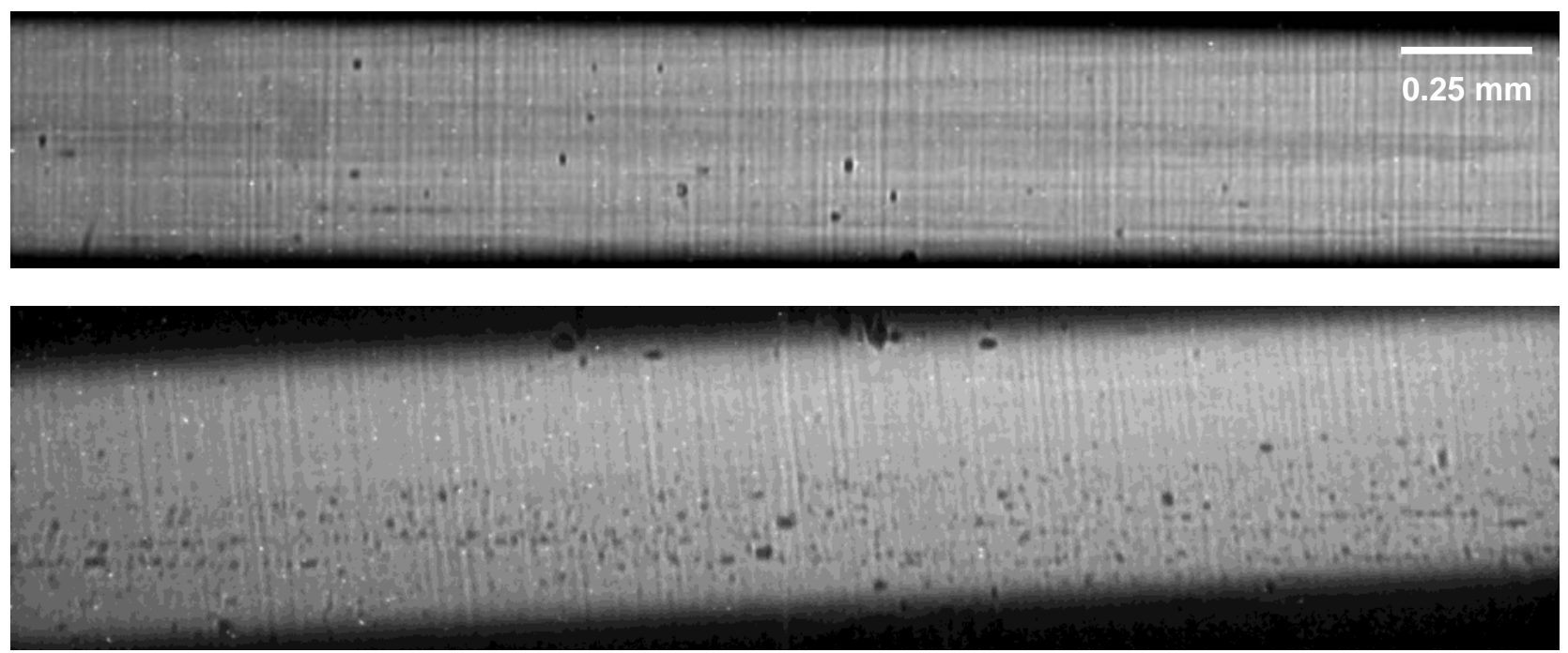

Figure 2. Images of wave fronts acquired after reflection on a $\mathrm{Mo} / \mathrm{Si}$ multilayer mirror (top) and $\mathrm{Pd} / \mathrm{B}_{4} \mathrm{C}$ mirror (bottom, both $2.5 \mathrm{~nm} \mathrm{~d}$-spacing, further details on the mirrors and coating parameters are published) ${ }^{6}$ at the tomography beamline 8.3.2 of the Advanced Light Source (USA) ${ }^{18}$. Note that a horizontal reflecting geometry was used while the images were rotated by 90 degree for the reproduction in this article. The vertical line pattern (actually horizontal) is present in both pictures and related to the beamline's exit window made from beryllium (cf. as well Fig. 1) as well as dark spots related to surface imperfections of both mirrors due to damages or contamination. Horizontal stripes are mainly present for the $\mathrm{Mo} / \mathrm{Si}$ multilayer due to wave front imperfections introduced by reflection on the multilayer, for $\mathrm{Pd} / \mathrm{B}_{4} \mathrm{C}$ the modulations are very weak related to the rather short mirror-detector distance.

\section{WHITE-LIGHT INTERFEROMETRY}

The surface roughness of reflective synchrotron optics is usually measured using an interferometric microscope (often referred to as white-light interferometer (WLI)). A Micromap Promap 512 was applied to investigate mirrors used in the initial study $\left(\mathrm{Mo} / \mathrm{Si} \text { and } \mathrm{Pd} / \mathrm{B}_{4} \mathrm{C} \text { as in the previous section }\right)^{6}$. Such instruments are commercial available and have been described in the literature ${ }^{19}$. The focus of interest is set on the mid-spatial frequency domain. The samples have been measured with the WLI in a Michelson-interferometer configuration of magnification $2.5 \times$ which covers a measurement area of $1.882 \mathrm{~mm}^{2}$. This allows one to characterize the micro-roughness on a spatial frequency range from $1882 \mu \mathrm{m}^{-1}$ to $6 \mu \mathrm{m}^{-1}$. Fig. 3 shows the measurement results for the micro-roughness in comparison to a super polished Si-substrate as well as pictures of the corresponding wave fronts acquired at ESRF beamline ID19 (details on the applied protocol are published $)^{10,14,20,21}$. Mainly on the $\mathrm{Pd} / \mathrm{B}_{4} \mathrm{C}$ coating a significant decrease of the micro-roughness $(0.69 \mathrm{~nm} \mathrm{rms})$ of factor 8 is found while the $\mathrm{Mo} / \mathrm{Si}(0.16 \mathrm{~nm} \mathrm{rms})$ shows a factor of two decrease compared to the uncoated $\mathrm{Si}(0.08 \mathrm{~nm} \mathrm{rms})$. Beside the higher value for the micro-roughness the measurements on the $\mathrm{Pd} / \mathrm{B}_{4} \mathrm{C}$ shows dots statistically distributed on the sample as well as streaks which can be assumed both to have an impact on the optical as well as wave front preservation properties. The slightly higher roughness on the $\mathrm{Mo} / \mathrm{Si}$ is to be assumed to cause mainly scattering only. It is worth to note that due to the X-ray reflectivity measurements published in frame of the original study the roughness of all mirrors is supposed to be similar. Hence, WLI for the first time delivers an indicator that the mirrors have different 
roughness properties ${ }^{6}$. A rough mirror can act as mild diffuser which can for example also lead to a more homogeneous wave front as outlined in the next section of this proceedings article.

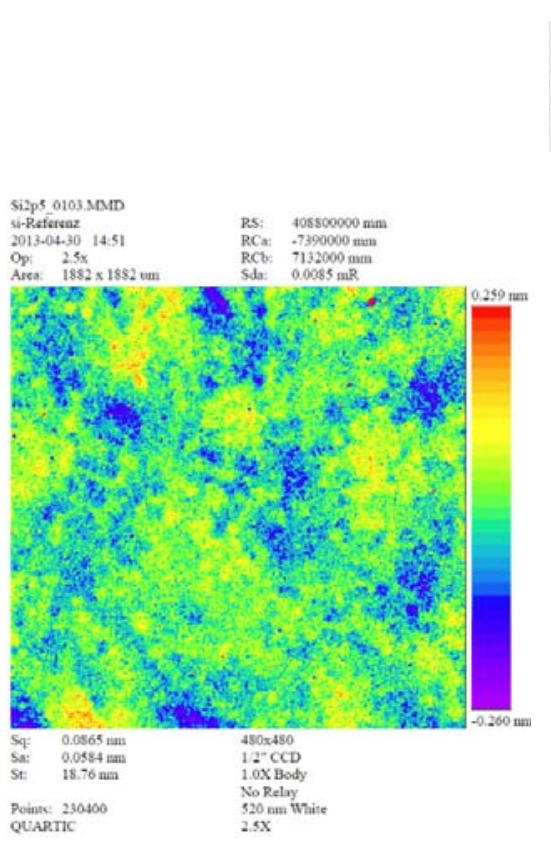

Si substrate (blank)
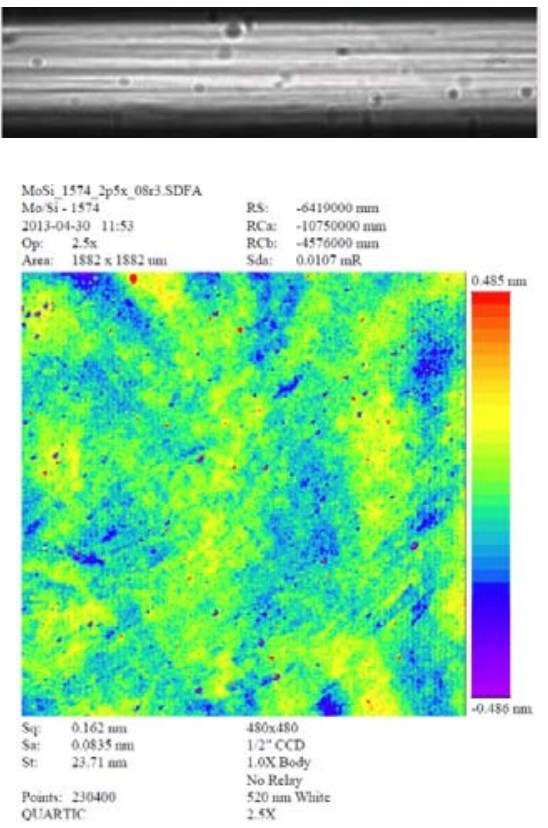

$\mathrm{Mo} / \mathrm{Si}$
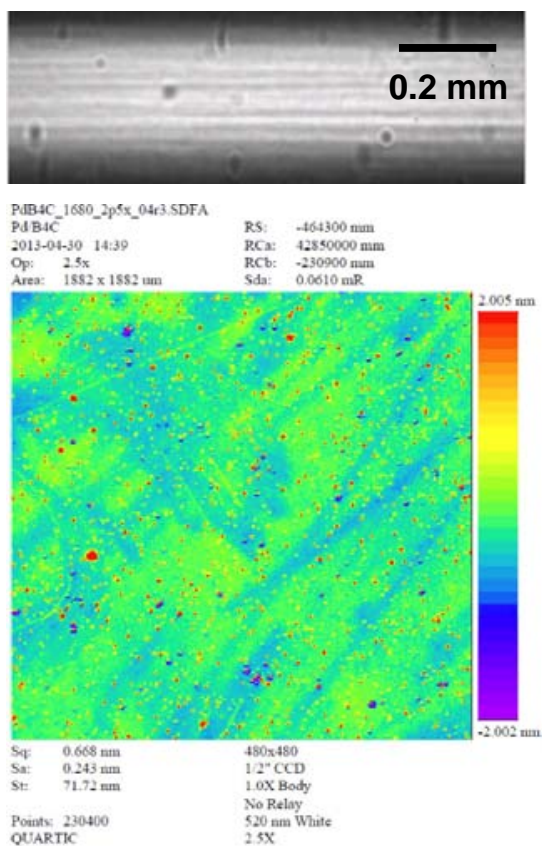

$\mathrm{Pd} / \mathrm{B}_{4} \mathrm{C}$

Figure 3. Top: wave fronts after reflection on the corresponding multilayer ( $2.5 \mathrm{~nm} \mathrm{~d}$-spacing) as measured at beamline ID19 $(\mathrm{ESRF})^{10,14}$, round objects visible are related to imperfections of the beamline's exit window made from $\mathrm{Be}^{23}$, bottom: WLI results of the indicated mirrors as well as of blank super-polished $\mathrm{Si}$ substrate. On the $\mathrm{Pd} / \mathrm{B}_{4} \mathrm{C}$ coating a significant decrease of the micro-roughness is found while the $\mathrm{Mo} / \mathrm{Si}$ shows minor decrease compared to the uncoated $\mathrm{Si}$. $\mathrm{Pd} / \mathrm{B}_{4} \mathrm{C}$ shows dots statistically distributed on the sample as well as streaks as already indicated in the initial study ${ }^{6}$.

\section{THE OTHER MULTILAYER}

Frequently beam diffusers are used at synchrotron light sources in order to improve the homogeneity of the wave front ${ }^{23}$. The interlayer roughness of a deposited multilayer is assumed to act in a diffusing manner as well, i.e. can be the reason why reflection on a multilayer mirror can affect the coherence properties of a wave front stronger than for example in comparison with a crystal-based device ${ }^{6}$. In order to study the impact of the interlayer roughness on the wave front, a super-polished $\mathrm{Si}$ substrate was partially coated with a W/B ${ }_{4} \mathrm{C}$ stripe $(120$ bi-layers, $2 \mathrm{~nm}$ d-spacing) according to parameters published before at the ESRF multilayer deposition facility ${ }^{13,17}$. Next to this stripe a second multilayer stripe was deposited, same parameters while the pressure in the coating facility was increased from $1 \mu$ bar to $5 \mu$ bar leading to an increased interlayer roughness (a third stripe deposited at $10 \mu$ bar was found to be poorly reflecting and not useful for imaging at all). The corresponding characterization is depicted in Fig. 4: on the left-top the wave front as acquired at beamline ID19 (ESRF) and the corresponding coherence characterization by means of Talbot imaging ${ }^{14}$ below (characterizations by means of X-ray reflectivity scans are depicted on the bottom of Fig. 4). Stripe pattern and coherence properties are similar to other multilayer mirrors previously studied in the same manner ${ }^{14}$. On the right the wave front for the reflection on the multilayer deposited at $5 \mu$ bar is shown as well the Talbot visibility study (the dspacing is $1.78 \mathrm{~nm}$ according to X-ray reflectivity characterization): while the coherence is only minor affected by the increased interlayer roughness of the multilayer the intensity of the stripe pattern, i.e. for example the peak-to-valley intensity between the dark and the bright stripes is substantially reduced. Furthermore, high-spatial frequency stripe components are less present. Commonly, such a wave front can be considered as better-suited for a simple flat field correction with respect to the wave front shown on the left. The coherence properties are sufficient to perform inline phase-contrast X-ray imaging by means of a propagation-based approach in combination with single-distance phaseretrieval techniques ${ }^{7}$. 

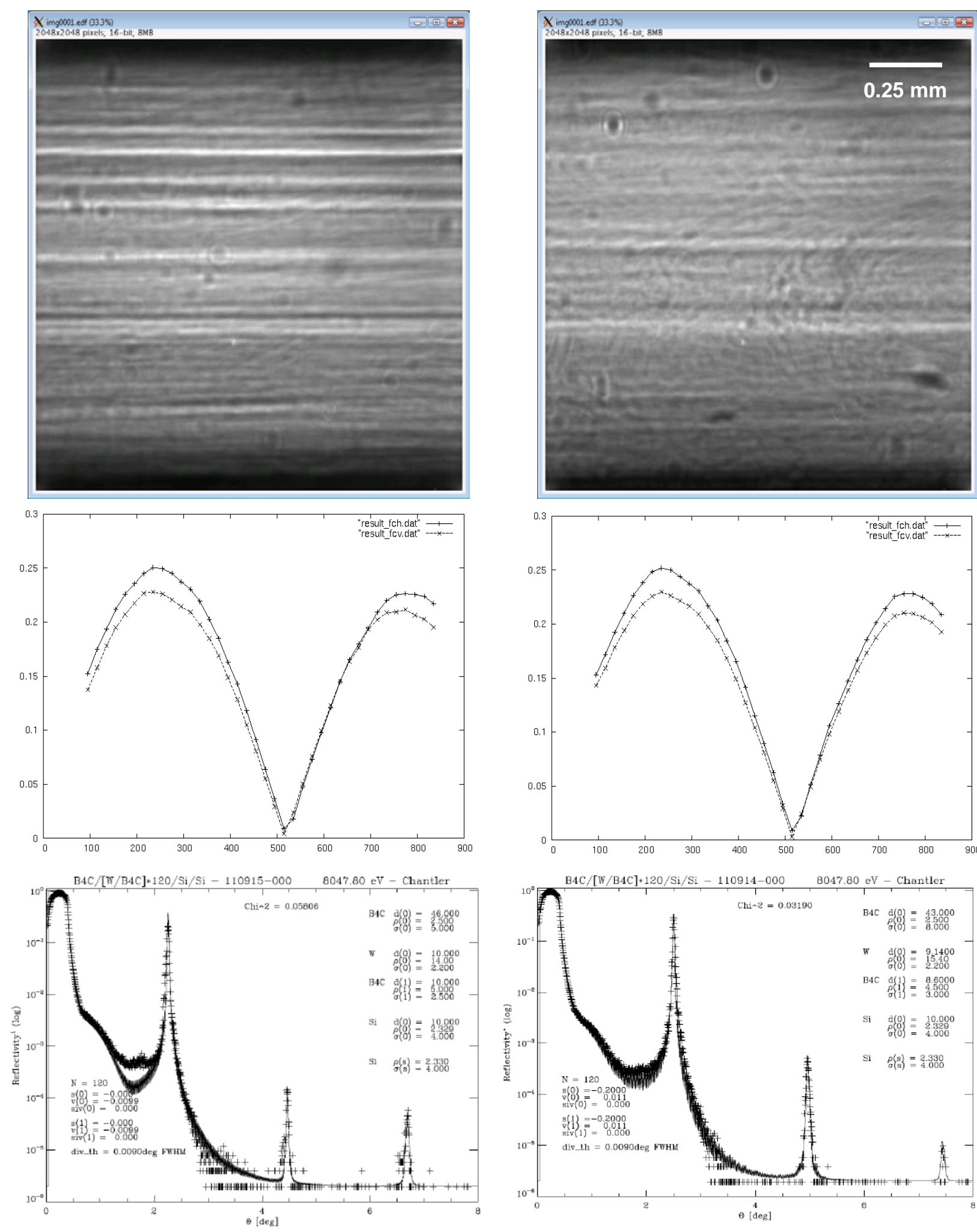

Figure 4. Top: wave fronts after reflection on W/ $\mathrm{B}_{4} \mathrm{C}$ multilayers coated at the ESRF multilayer deposition facilty (left: 120 bi-layers, $2 \mathrm{~nm}$ d-spacing, $1 \mu$ bar, right: 120 bi-layers, $1.78 \mathrm{~nm}$ d-spacing, $5 \mu$ bar) acquired at beamline ID19 (ESRF) ${ }^{10,14}$, middle: corresponding coherence measurements using Talbot imaging ${ }^{14,15}$, bottom: X-ray reflectivity characterization. 


\section{SUMMARY \& OUTLOOK}

The various studies carried out in recent years have demonstrated the need to further deepen our understanding on how the reflection on a multilayer mirror affects the properties of the reflected wave front. The origin of the wave front inhomogeneities, i.e. the irregular stripe pattern can be addressed to surface imperfections of the substrate. To verify this hypothesis currently a Si substrate specially polished to reduce shape errors has been obtained and is going to be coated with a standard multilayer deposition ${ }^{24}$. In order to explain in detail the difference wave fronts reported, numerical modeling of (partially) coherent wave fronts on (rough) multilayer coatings will be required.

\section{REFERENCES}

[1] Banhart, J. (ed.), "Advanced Tomographic Methods in Materials Research and Engineering", Oxford University Press (2008).

[2] Stock, S. R., "MicroComputed Tomography: Methodology and Applications", CRC Press, London, New York, Boca Raton (2008).

[3] Rack, A., Zabler, S., Müller, B. R., Riesemeier, H., Weidemann, G., Lange, A., Goebbels, J., Hentschel, M., Görner, W., "High resolution synchrotron-based radiography and tomography using hard X-rays at the BAMline (BESSY II)", Nucl. Instr. Meth. Phys. Res. A 586, 327-344 (2008).

[4] Rack, A., Weitkamp, T., Bauer Trabelsi, S., Modregger, P., Cecilia, A., dos Santos Rolo, T., Rack, T., Haas, D., Simon, R., Heldele, R., Schulz, M., Mayzel, B., Danilewsky, A. N., Waterstradt, T., Diete, W., Riesemeier, H., Müller, B. R., Baumbach, T., "The micro-imaging station of the TopoTomo beamline at the ANKA synchrotron light source", Nucl. Instr. \& Meth. in Phys. Res. B 267, 1978-1988 (2009).

[5] Rack, A., Riesemeier, H., Vagovic, P., Weitkamp, T., Siewert, F., Dietsch, R., Diete, W., Bauer Trabelsi, S., Waterstradt, T., Baumbach, T., "Fully automated, fixed exit, in vacuum double-multilayer monochromator for synchrotron-based hard X-ray micro-imaging applications," AIP Conf. Proc. 1234, 734-737 (2010).

[6] Rack, A., Weitkamp, T., Riotte, M., Grigoriev, D., Rack, T., Helfen, L., Baumbach, T., Dietsch, R., Holz, T., Krämer, M., Siewert, F., Meduna, M., Cloetens, P., Ziegler E., "Comparative study of multilayers used in monochromators for synchrotron-based coherent hard X-ray imaging," J. Synch. Rad. 17(4), 496-510 (2010).

[7] Weitkamp, T., Haas, D., Wegrzynek, D., Rack, A.., "ANKAphase: software for single-distance phase-retrieval from inline X-ray phase contrast radiographs," J. Synchrotron Radiat. 18 (4), 617-629 (2011).

[8] Rack, A., Helwig, H.-M., Bütow, A., Rueda, A., Matijasevic-Lux, B., Helfen, L.., Goebbels, J., Banhart J., "Early pore formation in aluminium foams studied by synchrotron-based microtomography and 3-D image analysis," Acta Mater. 57 (16), 4809-4821 (2009).

[9] Mochales, C., Maerten, A., Rack, A., Cloetens, P., Mueller, W. D., Zaslansky, P., Fleck, C., "Monoclinic phase transformations of zirconia-based dental prostheses, induced by clinically practised surface manipulations," Acta Biomater. 7 (7), 2994-3002 (2011).

[10] Weitkamp, T., Tafforeau, P., Boller, E., Cloetens, P., Valade, J.-P., Bernard, P., Peyrin, F., Ludwig, W., Helfen, L., Baruchel, J., "Status and evolution of the ESRF beamline ID19," AIP Conf. Proc. 1221, 33-38 (2010).

[11]Rack, A., Weitkamp, T., Zanette, I., Morawe, Ch., Vivo, A., Tafforeau, P., Cloetens, P., Ziegler, E., Rack, T., Cecilia, A., Vagovic, P., Harmann, E., Dietsch, R., Riesemeier, H., "Coherence preservation and beam flatness of a single-bounce multilayer monochromator (beamline ID19 - ESRF)," Nucl. Instr. Meth. Phys. Res. A 649 (1), 123-127 (2011).

[12] Rack, A., Assoufid, L., Dietsch, R., Weitkamp, T., Bauer Trabelsi, S., Rack, T., Siewert, F., Krämer, M., Holz, Th., Zanette, I., Lee, W.-K., Cloetens, P., Ziegler, E., "Study of multilayer-reflected beam profiles and their coherence properties using beamlines ID19 (ESRF) and 32-ID (APS)," AIP Conf. Proc. 1437, 15-17 (2012).

[13] Rack, A., Assoufid, L., Lee, W.-K., Shi, B., Liu, C., Morawe, Ch., Kluender, R., Conley, R., Bouet, N., "Hard $\mathrm{X}$-ray multilayer mirror round-robin on the wavefront preservation capabilities of W/B4C coatings," Radiat. Phys. \& Chem. 81 (11), 1696-1702 (2012).

[14] Rack, A., Weitkamp, T., Assoufid, L., Rack, T., Zanette, I., Morawe, Ch., Kluender, R., David, C., "Protocol to study wavefront preservation capabilities of reflective X-ray optics with coherent synchrotron light," Nucl. Instr. Meth. Phys. Res. A 710C, 100-104 (2013).

[15]Kluender, R., Masiello, F., van Vaerenbergh, P., Härtwig, J., "Measurement of the spatial coherence of synchrotron beams using the Talbot effect," phys. status solidi A 206(8), 1843-1845 (2009). 
[16] Morawe, Ch., Barrett R., Friedrich, K., Klünder, R., Vivo, A., “Spatial coherence studies on x-ray multilayers, “ Proc. of SPIE 8139, 813909 (2011).

[17] Morawe, Ch., Borel, Ch., Peffen, J.-Ch., “The new ESRF multilayer deposition facility,” Proc. of SPIE 6705, 670504 (2007).

[18] MacDowell, A.A., Parkinson D.Y., Haboub, A., Schaible, E., Nasiatka, J.R., Yee, C.A., Jameson, J.R., AjoFranklin, J.B., .Brodersen, C.R., McElrone, A.J., "X-ray micro-Tomography at the Advanced Light Source" in Developments in X-Ray Tomography VIII, edited by Stuart R. Stock, Proceedings of SPIE Vol. 8506 (SPIE, Bellingham, WA, 2012), 8506-18.

[19] Wyant, J. C., "White light interferometry," Proc. SPIE 4737, 98-107 (2002).

[20] Douissard, P.-A., Cecilia, A., Martin, T., Chevalier, V., Couchaud, M., Baumbach, T., Dupré, K., Kühbacher, M., Rack, A., "A novel epitaxically grown LSO-based thin-film scintillator for micro-imaging using hard synchrotron radiation,” J. Synchrotron Radiat. 17 (5), 571-583 (2010).

[21] Douissard, P.-A., Cecilia, A., Rochet, X., Chapel, X., Martin, T., van de Kamp, T., Helfen, L., Baumbach, T., Luqout, L., Xiao, X., Meinhardt, J., Rack, A., "A versatile indirect detector design for hard X-ray microimaging,“ J. Instrum. 7 (9), P09016 (2012).

[22] Morgan, K. S., Irvine, S. C., Suzuki, Y., Uesugi, K., Takeuchi, A., Paganin, D. M., Siu, K. K. W., "Measurement of hard X-ray coherence in the presence of a rotating random-phase-screen diffuser," Opt. Comm. 283 (2), 216-225 (2010).

[23] Espeso, J.I., Cloetens, P., Baruchel, J., Härtwig, J., Mairs, T., Biasci, J.-C., Marot, G., Salomé-Pateyron, M., Schlenker, M., "Conserving the Coherence and Uniformity of Third-Generation Synchrotron Radiation Beams: the Case of ID19, a 'Long' Beamline at the ESRF," J. Synchrotron Radiat. 5 (5), 1243 (1998).

[24] Yamauchi, K., Yamamura, K., Mimura, H., Sano, Y., Saito, A., Endo, K., Souvorov, A., Yabashi, M., Tamasaku, K., Ishikawa, T., Mori, Y., "Wave-optical evaluation of interference fringes and wavefront phase in a hard-x-ray beam totally reflected by mirror optics," Appl. Optics 44 (32), 6927-6932 (2005).

[25] Tromba, G., Longo, R., Abrami, A., Arfelli, F., Astolfo, A., Bregant, P., Brun, F., Casarin, K., Chenda, V., Dreossi, D., Hola, M., Kaiser, J., Mancini, L., Menk, R. H., Quai, E., Quaia, E., Rigon, L., Rokvic, T., Sodini, N., Sanabor, D., Schultke, E., Tonutti, M., Vascotto, A., Zanconati, F., Cova, M., Castelli, E., "The SYRMEP Beamline of Elettra: Clinical Mammography and Bio - medical Applications," AIP Conf. Proc. 1266, 18 (2010). 